elements need to also to be much improved, since they are crucial for our understanding of various important nucleosynthetic processes in stars.

We have shown that the atmospheres of CP stars can be used to assess the new photoionization data provided by OP. Such an assessment is essential in view of the larger opacities obtained by $\mathrm{OP}$, which have allowed to solve several key problems in stellar astrophysics.

Nahar, S.N. \& Pradhan, A.K. 1994, J. Phys. B, 27, 429

Pradhan, A.K. \& Berrington, K.A. 1993, J. Phys. B, 26, 157

Raymond, J.C. 1988, in Pallavicini, R., ed, Hot Thin Plasmas in Astrophysics, Kluwer Ac.

Publ. Dordrecht, p.3

Smith, B.W., Raymond, J.C., Mann, J.B., Jr. \& Cowan, R.D. 1985, ApJ, 298, 898

\title{
THE OPACITY DRIVEN PULSATORS
}

\author{
WOJCIECH A. DZIEMBOWSKI \\ Copernicus Astronomical Center, ul. Bartycka 18, \\ 00-716 Warszawa, Poland
}

The opacity mechanism is the only well understood excitation mechanism of observed stellar oscillations. The driving effect arises due the a specific form of the opacity perturbation which leads to a conversion of the radiative flux energy into kinetic energy of oscillations. In 1963 Baker and Kippenhan demonstrated that the opacity mechanism acting in the HeII ionization zone is the cause of the Cepheid pulsations. Subsequently it has been shown that the same mechanism works in a large variety of stars. There are three opacity bumps that play a role in pulsation excitation in various types of objects - one connected with the $\mathrm{H}$ ionization, one connected with $\mathrm{He}$ II ionization zone - and one, discovered quite recently by Iglesias, Rogers \& Wilson (1988), caused by a large agglomeration of metal element (mostly $\mathrm{Fe}$ ) lines.

I focus on Main Sequence pulsators. There is a considerable difference among these stars in the manifestation of pulsation instability. However in all four types of Main Sequence pulsators - ro (rapidly oscillating) Ap, $\delta$ Scuti, $\beta$ Cephei, and SPB stars - a common feature is their multiperiodic variability. FG Vir a $\delta$ Scuti, having 10 modes definitely detected (Breger (1984)), is the best object in the class.

The observed variability in roAp stars is caused by excitation of high-order p-modes with frequencies similar to those of solar oscillation. The amplitudes, however, are by three orders of magnitude higher. The excitation is most likely due to the opacity bump in the H I 
ionization zone, but magnetic fields play an important role in mode selection. Studies of these stars may help us to understand the nature of Ap stars in general.

In $\delta$. Scuti star models, unstable modes cover a wide range of frequencies around the fundamental radial mode frequency. The instability extends to high spherical harmonic degrees. The driving effect is essentially the same as in the classic pulsating variables. Unstable modes include low order $g$-modes which probe very well the $\mu$-gradient surrounding the convective core. Treatment of such modes remains subject of controversy.

The origin of pulsation in $\beta$ Cephei stars has been an outstanding puzzle for stellar stability theory. The improved Iglesias \& Rogers (1992) data lead to a fully satisfactory solution in terms of an opacity mechanism acting in the metal bump zone. The unstable modes are similar to those in $\delta$ Scuti stars though the instability does not extend to p-modes of higher order than $p_{1}$ or $p_{2}$. Studies of $\beta$ Cephei star pulsation have the potential for probing the interiors of massive stars.

The SPB stars are a newly defined group of pulsating stars (Waelkens 1992) containing Main Sequence objects in the spectral type range B3 - B8 which are variable with periods from 0.5 to 4 days. The observational evidence for $\mathrm{g}$-mode excitation was quite convincing. Subsequently it has been shown that the same mechanism that causes pulsations in $\beta$ Cephei stars in less massive objects drives high-order g-modes with periods consistent with those determined in SPB stars. These modes are excellent probes of the whole radiative interior, however the much longer period presents a very different challenge for observers than in the case of the other types of pulsating Main Sequence stars.

\title{
ATMOSPHERES AND WINDS OF HOT STARS - IMPACTS OF NEW OPACITY CALCULATIONS AND CONTINUING NEEDS
}

\author{
KEITH BUTLER \\ Universitäts - Sternwarte München, Scheinerstr. 1, 81679 München, Germany
}

In this paper I review some recent advances in the use of large amounts of atomic data in the modelling of atmospheres and winds of hot stars. The review is highly selective but representative of current developments. A more general overview is to be found in Kudritzki and Hummer (1990) although the field is changing so rapidly that much has happened since then. The paper breaks down into three parts: work on line formation, in which the atmospheric structure is known and held fixed, is described first, then follows a description of the inclusion of line opacities in non-LTE in the atmosphere problem itself, and finally recent developments in the theory of radiatively driven stellar winds are summarized. Here special emphasis is given to a novel distance determination method based 